\title{
Spinning Equipment Using Air-Flow
}

\author{
By Yukio Urano, Mitsunori Horiuchi, Toshio Samata \\ and Shinichi Hamabata, Members, TMSJ
}

Kanegafuchi Spinning Co., Ltd. Osaka

\begin{abstract}
This article reports on a new type of spinning equipment which, using air flow, differs radically in the method of twisting from the ring spinning frame and which needs no ring, traveller or pot.

By this continuous spinning equipment, yarns, after spun with rovings can be wound directly into the cheese.

The equipment is capable of a spindle speed of more than 20,000 r.p.m. The yarn spun by this equipment- "air-spun yarn," as we call it-has some defects, particularly in strength and elongation, when compared with ring-spun yarn.
\end{abstract}

\section{Introduction}

Various ways have been tried to speed-up fine spinning frames and to dispense with the rewinding process. The United States, Czechoslovakia and West Germany ${ }^{1,2,3)}$ have come out with spinning equipment which uses air flow. In Japan, too, similar equipment has been devised and is mentioned in patent news. ${ }^{4}$ )

Research conducted by the present authors since February, 1959 has given birth to what we call "air spinning equipment". It is the so-called open-end system. It cuts off, by using air flow, the continuity of fiber tufts during drafting and spins yarn without the continuity of fiber tufts.

Use of air flow, however, is accompanied by various problems, and yarn quality leaves room for improvement.

\section{Process of Research}

We will begin by explaining in what part of the air spinning equipment air flow is used. The equipment is featured by its method of twisting.

As shown in Fig. 1(a), the fiber tuft nipped at $\mathrm{A}$ and $\mathrm{B}$ is turned at $\mathrm{C}$ to produce the same number of twists in opposite directions between $\mathrm{A}$ and $\mathrm{C}$ between $B$ and $C$. Turn $A$ and $B$ so as to shift the fiber tuft from $B$ to $A$, and the twists between $A$ and $C$ offset those between $B$ and $C$. The fiber tuft drawn from A will have no twists left on it.

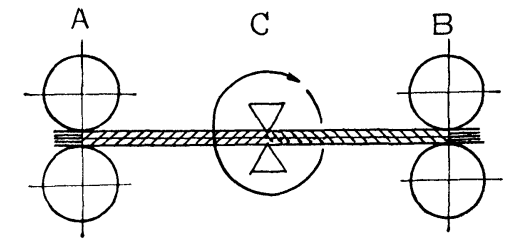

(a)

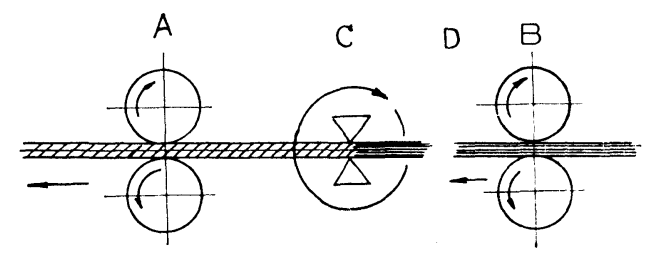

(b)

Fig. 1 Where air-flow is used

As shown in Fig. 1(b), the fiber tuft is nipped by rollers $A$ and $B$ and shifted to the left, while at $\mathrm{C}$ it is turned. At $\mathrm{D}$ the continuity of fiber tufts is cut off so that there will be no twists formed between $\mathrm{B}$ and $\mathrm{C}$, and fiber is again continuously supplied to $C$. Twists are formed only between $\mathrm{A}$ and $\mathrm{C}$. The yarn can, therefore, be continuously drawn from A. Here air-flow is used to do its work at D.

\section{2-1 Equipment}

Fibers are beaten and opened by the fan, guided by the air current through the introducing pipe into 
the cylinder and blown along the direction tangent to the spindle. Moreover, they are carried by the air current to circulate around the cylinder until they are assembled in the center of the cap, where they stick to the preceding fiber tuft. The fiber tuft is twisted into yarn between the twister (made of a plate spring and used to nip the fiber tuft) and the roller. The twister is fixed to, and turns with, the spindle.

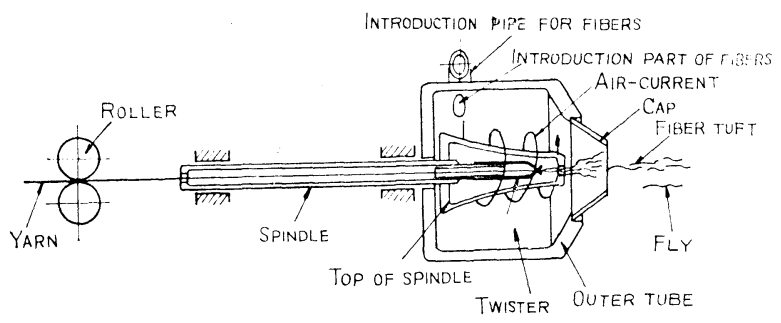

Fig. 2 Trial equipment in early stage

This equipment had many defects, notably (1) a large fiber loss because fibers could not stick to the preceding fiber tuft and were blown out, as fly, to the outside, (2) the formation of a large number of knobs and (3) frequent occurrence of yarn breakage because of the spiral rotation of the tip of spindle by the air current.

\section{2-2. Equipment in Present Stage}

The equipment, even in its early stage, was capable of spinning yarn, but it was not useful in the study of yarn quality. Figs. 3 and 4 show the improved type of this equipment.

Fibers of roving are fed into the fan by the feed rollers, beaten and pulled off by the blades of the

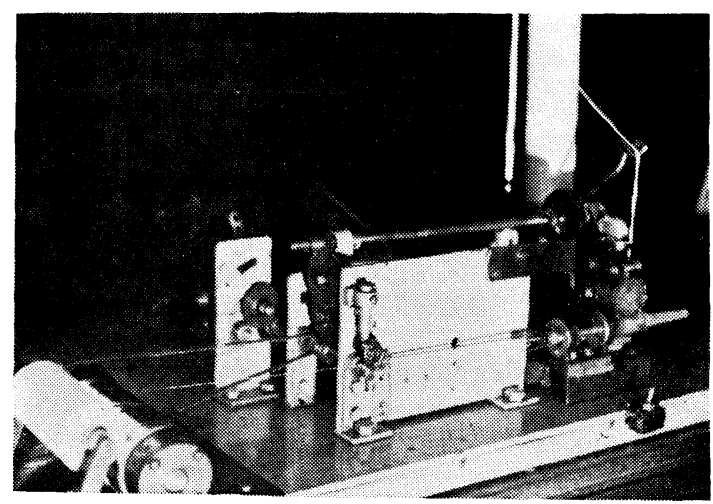

Fig. 3 Trial equipment for the air-spinning system using the air flow fan and carried away by the air current to stick to the preceding fiber tuft one after another.

This air current escapes to the outside through the small hole in the cap, and the fiber tuft is pulled out by the front rollers. On its way, the tuft is twisted between the twister and the front rollers by the turning of the twister fixed to the spindle. The fiber tuft drawn out by the front rollers is, therefore, already a yarn and can be wound directly into the cheese.

At the start, it will do to draw yarn by inserting a specially designed wire into the cap in the direction opposite to the direction in which the yarn is moving, and sticking the yarn to the fibers which are carried by the air current from the fan and pulling it out. An experiment along this line was made at a spindle speed of 20,000 r.p.m. and a fan speed of 16,000 r.p.m.

\section{2-3. Reconstruction of the Twister}

The number of twists per inch of the yarn spun by using the twister shown in Fig. 4 is 34.5 t/in mathematically, as against the actual $18.0 \mathrm{t} / \mathrm{in}$.

Fig. 5 is a photo showing the appearance of the twisting state of air-spun yarn (see Fig. 4). Rather

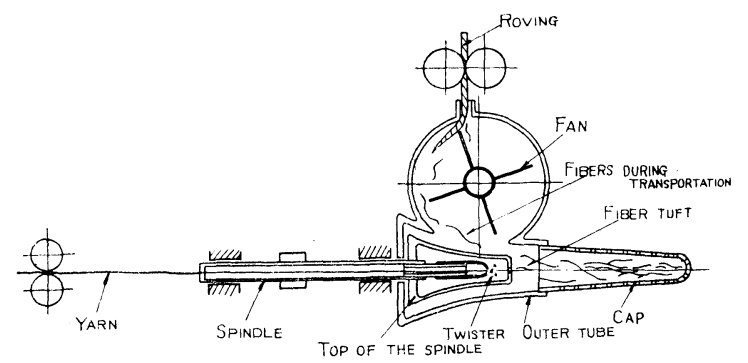

Fig. 4 Trial equipment for the air spinning system using the air flow

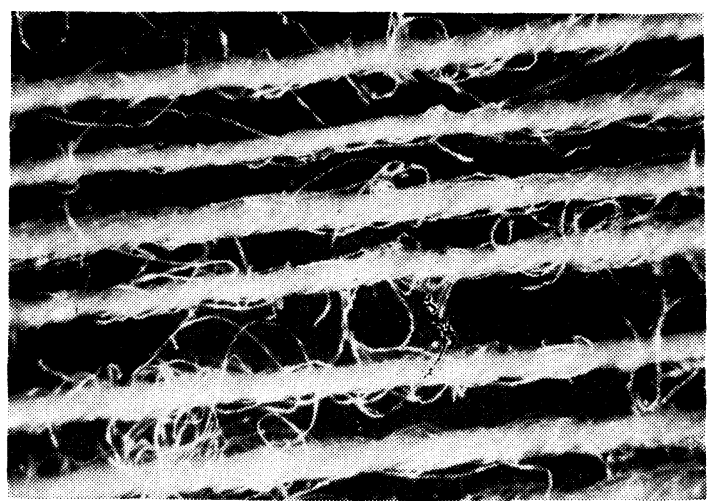

Fig. 5 Appearance of yarn spun by the trial equipment shown in Fig. 4

Journal of The Textile Machinery Society of Japan 
hard twists are found in the outer portion.

The photo in Fig. 6 shows the state of air-spun yarn with its twists untwisted. No twists are found in the central axis of the yarn, although some twists still remain in the outer portion. This shows that it is rather difficult to twist the central portion of yarn, although it is easy to twist the outer portion.

This fact presumably results from a decrease in the nipping force of the twister due to centrifugal force.

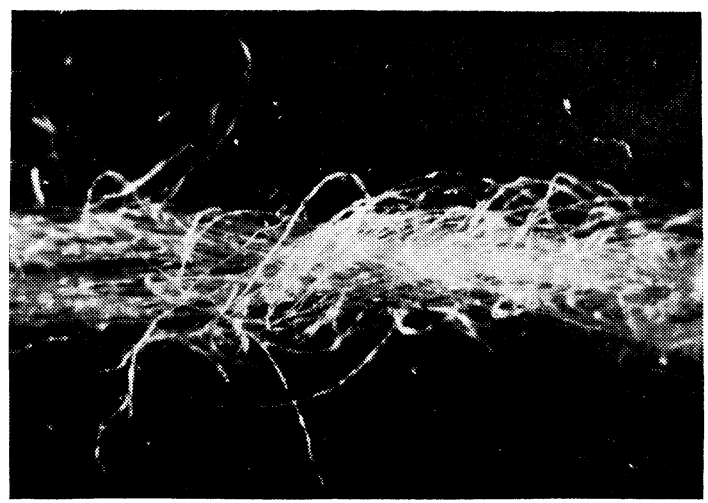

Fig. 6 Yarn shown in Fig. 5 with its twists untwisted to some extent

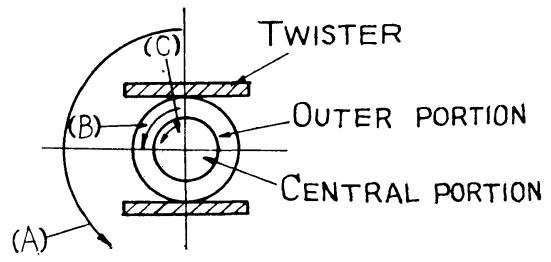

Fig. 7 Schematic diagram explaining the nipping of the fiber tuft by the twister

As is clearly explained in Fig. 7, a fiber tuft is nipped by the elasticity of the twister, which centrifugal force tends to reduce. Let $\mathrm{A}$ be the amount of turning of the twister. Slippage between the twister and the outer portion of the fiber tuft reduces the amount of turning until it is B. The inner portion of the fiber tuft delays the turning of the twister even more than does the outer portion until the amount of turning becomes $\mathrm{C}$.

This understanding of the twisting mechanism helps to explain the difference between the calculate number of twists per inch and the actual number and also the softness of the twists in the center of the fiber tuft.

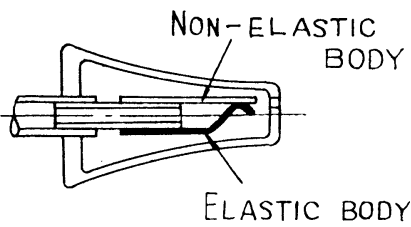

Fig. 8 Modified twister

Fig. 8 shows a twister which is hardly affected by centrifugal force. The difference between this twister and the twister in Fig. 4 lies in the fact that the fiber-nipping point of the twister in Fig. 8 has shifted slightly from the center line, and that only one side of the twister is made of a spring. This alteration improves fiber tuft-nipping force and brings the actual number of twists per inch to the calculated number, as is shown in Table 1 . The reports hereinafter are based on results obtained by using the improved twister.

\section{Comparing Ring Spinning Frame and Trial Equipment}

\section{3-1 Difference in Mechanism}

The basic difference between the ring spinning frame and the trial equipment is in the method of twisting. The ring spinning frame twists by the rotation of the take-up part. The trial equipment twists by the rotation of the supplying part.

\section{3-1-1 Make-up of Spindle}

The path of yarn in the ring spinning frame wraps up the spindle. Therefore, the weight of the free end is increased, and the weight to be turned adds to itself the weight of the bobbin, thus making it easier to get an eccentric load.

Table 1 Comparison of Calculated Number of Twists per Inch by Calculation and Actual Number when Modified Twister is used.

\begin{tabular}{ccc}
\hline $\begin{array}{c}\text { Calculated number } \\
\text { of twists } \\
\text { (t/in) }\end{array}$ & $\begin{array}{c}\text { Actual number } \\
\text { of twists } \\
\text { (t/in) }\end{array}$ & Ratio \\
\hline 18.8 & 16.6 & $(\%)$ \\
20.4 & 16.8 & 88.4 \\
22.3 & 22.2 & 82.4 \\
24.6 & 23.2 & 99.5 \\
\hline
\end{tabular}


Thus, the spindle speed is limited by the way the bearing is built.

The traveller in a running ring spinning frame is easily burned by friction between the ring and the traveller, causing frequent traveller dispersions, which, too, restricts the speed within a certain limit. The trial equipment, on the contrary, helps to raise the spindle speed

\section{3-1-2 Packages compared}

The size of the package made by the ring frame is limited by the diameter of the ring. The trial equipment does winding independently of the twisting part and, therefore, is extremely useful for increasing the size of the package. The ring spinning requires the rewinding process. The trial equipment does direct cheese-winding.

\section{3-1-3 Mehtod of twisting}

With the ring spinning frame, the number of twists can be made exact by the rotation of the package. With the trial equipment, twists made by the rotation of the twister is not exact if the twister does not nip sufficiently.

\section{3-1-4 Draft}

Drafting in the ring spinning frame is done by the drafting rollers. Drafting in the trial equipment is done by pulling off the fibers from the tuft with the blades of the fan and sending away the fibers by the air current. Theorefore, drafting higher than that done by the roller-draft is possible. However, there still are many problems accompanying the operation of sending away fibers by the air current.

\section{3-2 Spun Yarns Compared}

By using rovings made from cotton 1 inch in fiber length, 20's yarns were spun on the ring spinning frame and the air-spinning equipment for comparison. Fig. 9 shows the ring-spun yarn; Fig. 10, the airspun yarn.

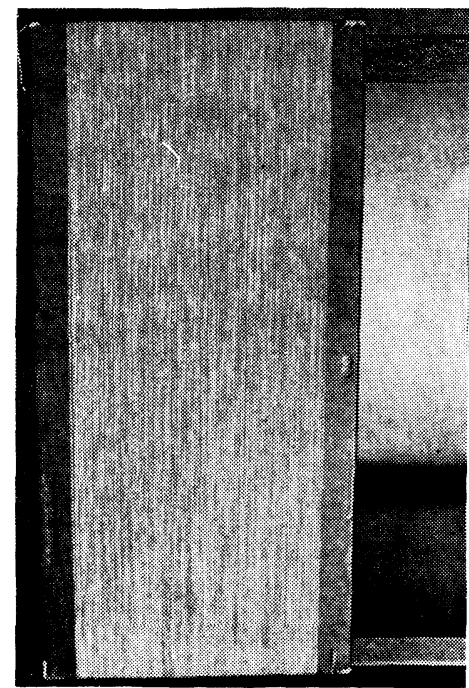

Fig. 9 Appearance of ring-spun yarn Count: 20.23's

No. of twists: $18.7 \mathrm{t} /$ in

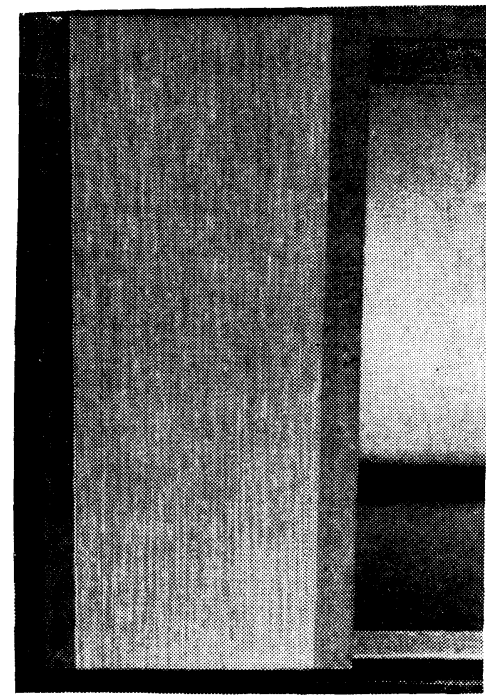

Fig. 10 Appearance of the air-spun yarn Count: 19.99's

No. of twists: $19.2 \mathrm{t} /$ in

Table 2 Results for Spun Yarn

\begin{tabular}{|c|c|c|c|c|c|c|}
\hline & $\begin{array}{l}\text { Count } \\
\text { (s) }\end{array}$ & $\begin{array}{l}\text { No. of } \\
\text { twists } \\
\text { (t/in) }\end{array}$ & $\begin{array}{c}\text { Variance of } \\
\text { yarn count } \\
(\%)\end{array}$ & $\begin{array}{c}\text { Average } \\
\text { strength } \\
\text { (lb) }\end{array}$ & $\begin{array}{l}\text { Variance } \\
\text { of yarn } \\
\text { strength } \\
(\%)\end{array}$ & $\begin{array}{c}\text { Elongation } \\
(\%)\end{array}$ \\
\hline Ring-spun yarn & 20.23 & 18.7 & 2.0 & 101.5 & 5.7 & 7.14 \\
\hline
\end{tabular}


No significant difference in evenness or the amount of neps is found between the ring-spun yarn and the air-spun yarn, except that the face of the air-spun yarn has a rough feel. Nor is a significant difference found between the two yarns in the variance of yarn count or the variance of yarn strength, although the air-spun yarn is weaker in strength and smaller in elongation. Generally speaking, the air-spun yarn is not yet equal in quality to the ring spun yarn. Table 2 shows the results on the two spun yarns.

\section{Investigating Defects}

The serious defects of air-spun yarn are its roughness, weaker strength and smaller elongation. These defects presumably arise from the beating of fibers by the fan, the sending of fibers by air current and the twisting mechanism of this equipment. Let us investigate these presumable causes.

\section{4-1 Damage of Fibers by the Blades of Fan}

We investigated by subjecting fibers to a Pressley strength test to find the degrees of their damage before and after passage through the fan. A $1.4 \%$ deterioration rate of fiber strength was noticed (Table 3 ), but this can not conceivably affect yarn strength.

Table 3 Pressley Strength

\begin{tabular}{ccc}
\hline $\begin{array}{c}\text { Before passage } \\
\text { through the fan } \\
\left(1 \mathrm{bs} / \mathrm{in}^{2}\right)\end{array}$ & $\begin{array}{c}\text { After passage } \\
\text { through the fan } \\
\left(1 \mathrm{bs} / \mathrm{in}^{2}\right)\end{array}$ & Ratio \\
\hline $70.8 \times 10^{3}$ & $69.8 \times 10^{3}$ & 98.6 \\
\hline
\end{tabular}

(Remarks) Sample is the roving for the 30's yarn

Breaking points caused by beating by the fan blades was checked with a staple diagram. Hardly any difference was found in the effective fiber length, except a slight increase in the rate of the shortfiber content. However, fiber breaking points were not big enough to contribute to a deterioration of yarn strength.

\section{4-2 Bending State of Fibers Constituting Spun Yarns}

Figs. 11 and 12 are photo showing the fiber tufts of ring-spun yarn and air-spun yarn completely untwisted.
The photos show that air-spun yarn has more bendings. To get the degree of bending of airspun yarn, we made staple diagrams of both ringspun and air-spun yarns by the following procedure:

1) About $80 \mathrm{mg}$ of each yarn cut exactly $15 \mathrm{~mm}$ long was prepared.

2) This sample was carefully untwisted without breaking any fibers.

3) Untwisted fibers were then made into staple diagrams.

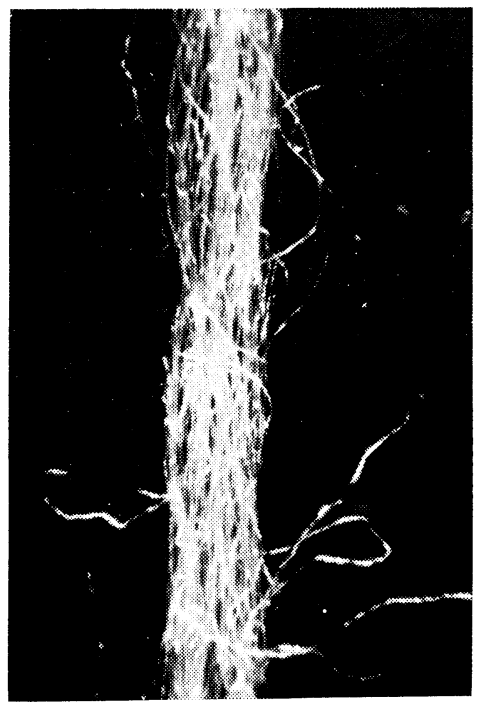

Fig. 11 Fiber tuft of ring-spun yarn completely untwisted

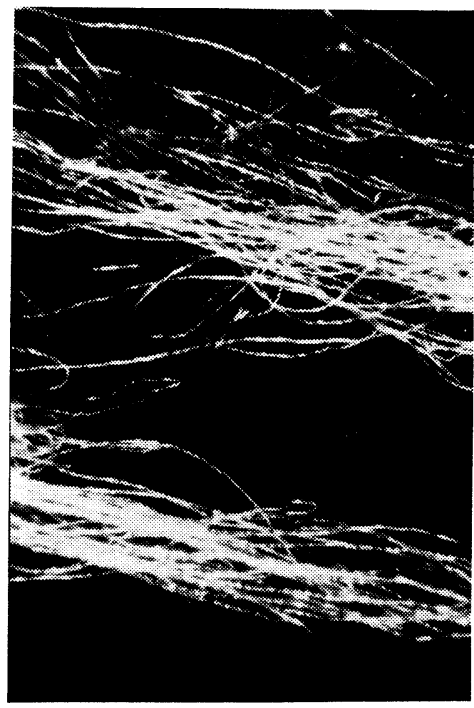

Fig. 12 Fiber tuft of air-spun yarn completely untwisted 
Staple diagrams made by this method are shown in Fig. 13.

(a) is for ring-spun yarn and (b) for air-spun yarn. If fibers constituting a yarn had no bending, the staple diagrams would have no fibers more than 15 $\mathrm{mm}$ long. The presence of fibers more than $15 \mathrm{~mm}$ long shows that fibers bend to that very extent. In (a), fibers more than $15 \mathrm{~mm}$ long are extremely few. In (b), about $45 \%$ of the fibers are more than $15 \mathrm{~mm}$ long. In other words, air-spun yarn has more bendings.

The presence of many fibers which bend is doubtless the reason for the weaker strength and the smaller elongation of yarn. The bendings of constituent fibers come from the fact that they are twisted in a shorter length than their real length.

\section{4-3. Investigating Transportation of Fibers by Air Current}

It is believed that fibers bend during their transportation by air current.

To investigate fiber transportation by air current, a transport tube $22 \mathrm{~mm}$ in diameter and $1 \mathrm{~m}$ long was fixed (in place of the cap in Fig. 4) and the behavior of fibers during air transportation was observed at a place $250 \mathrm{~mm}$. from the center of the fan. Figs. 14 and 15 are photos taken at fan speeds of 16,000 r.p.m. and 12,000 r.p.m. Fibers were transported in a very irregular condition. At 16,000 r.p.m. fibers were transported in a comparatively scattered condition. At 12,000 r.p.m. entanglements of fibers occurred. Fiber bendings during air transportation may be taken as the reason for inadequate yarn strength, the biggest defect in air-spun yarn. It calls for further research.

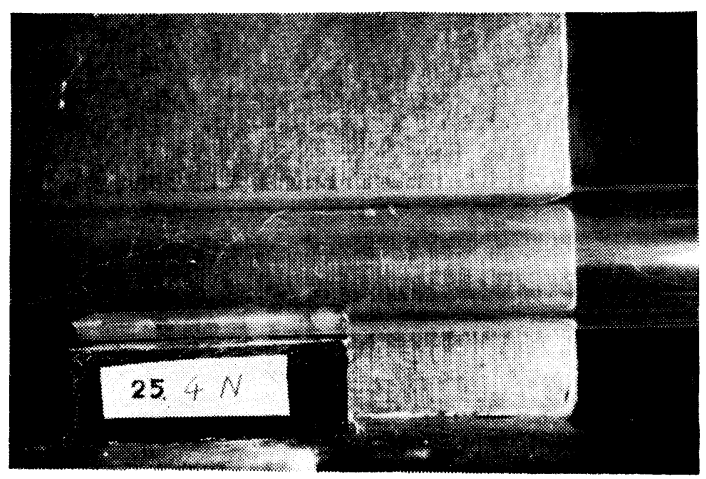

Fig. 14 Fibers during air transportation Distance from the center of the fan: $250 \mathrm{~m} / \mathrm{m}$ Fan speed: 16,000 rpm.

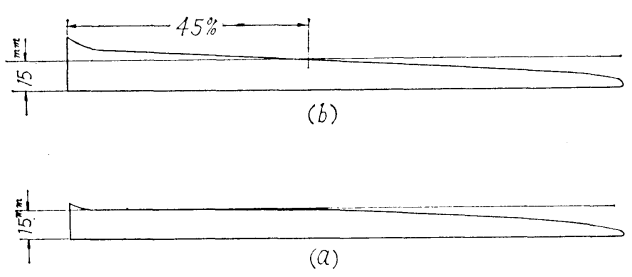

Fig. 13 Staple diagrams obtained by investigating the degree of fiber bending

\section{4-4. Feel of Yarn Face}

The face of air-spun yarn has a rough feel. This presumably is because of the twister. In the ring spinning frame which need not nip the fiber tuft, the fiber tuft is twisted by the traveller. In the trial equipment the fiber tuft is nipped and twisted by the twister. Therefore, not all fibers in the tuft are twisted in a uniform condition; fibers tend to get more twists on the outer portion. Moreover the entangled fibers inside the twister become neps, thus spoiling the appearance of the spun yarn.

\section{Conclusions}

The method of spinning by stopping the continuity of the fiber tuft once and then continuously supplying fibers by the use of air-flow has been successful. Although air-spun yarn compared with ring-spun yarn, still has a number of defects, it gives possibilities of realizing what cannot be hoped for from the conventional spinning frame: big package, high spindle speed and also dispensing with the rewinding process.

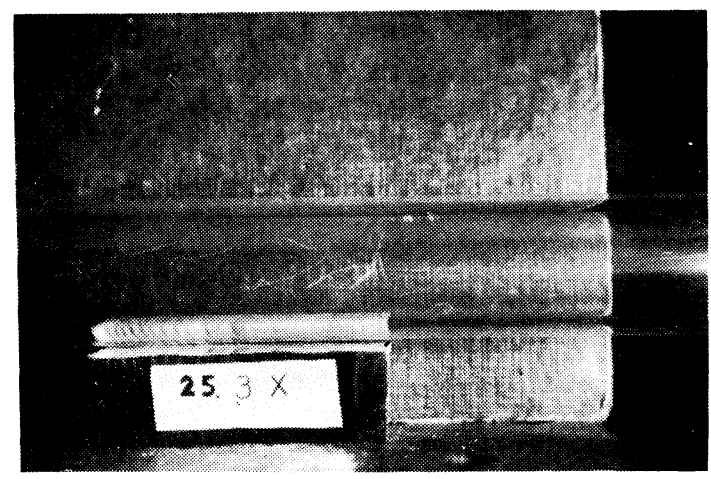

Fig. 15 Fibers during air transportation Distance from the center of the fan: $250 \mathrm{~m} / \mathrm{m}$ Fan speed: 12, $000 \mathrm{rpm}$.

Iournal of The Textile Machinery Society of Japan 
(1) In the trial equipment, which has no ring or traveller, spindle is easily supported and the spindle speed is also easily increased. Moreover, it can do directly winding into the cheese and can, therefore, omit the rewinding process.

(2) The strength and twist of air-spun yarn is not up to the standard of ring-spun yarn.

(3) Fiber damage due to beating by the blades of fan has no great effect on yarn strength.

(4) Deterioration in yarn strength comes from the fact that the yarn is spun from the fibers of a bent shape.

(5) The bending of fiber occurs when the fiber is transported by air flow from the fan.

(6) The rough feel of the yarn is traceable to some defect, at present unidentified, in the twisting mechanism.
Postscript: Patents Nos. 300986 and 401379 have been obtained for the trial equipment. Several other patents are pending.

We thank Dr. S. Watanabe and Dr. S. Fujii, professors at Tokyo University, and the members of its reasearch institute for their invaluable advice.

\section{Literature Cited :}

[1] George J. Kyame, Harbert R. Copeland; Text. World, 112, 70-73 (1962-5)

[2] P. Abbenheim; Text. Ind., 126, 109 (1962-5)

[3] The official report of the Patent Agency of Japan, 1959-674

[4] The official report of the Patent Agency of Japan. 1963-5865 